\title{
Habitability Models for Planetary Sciences
}
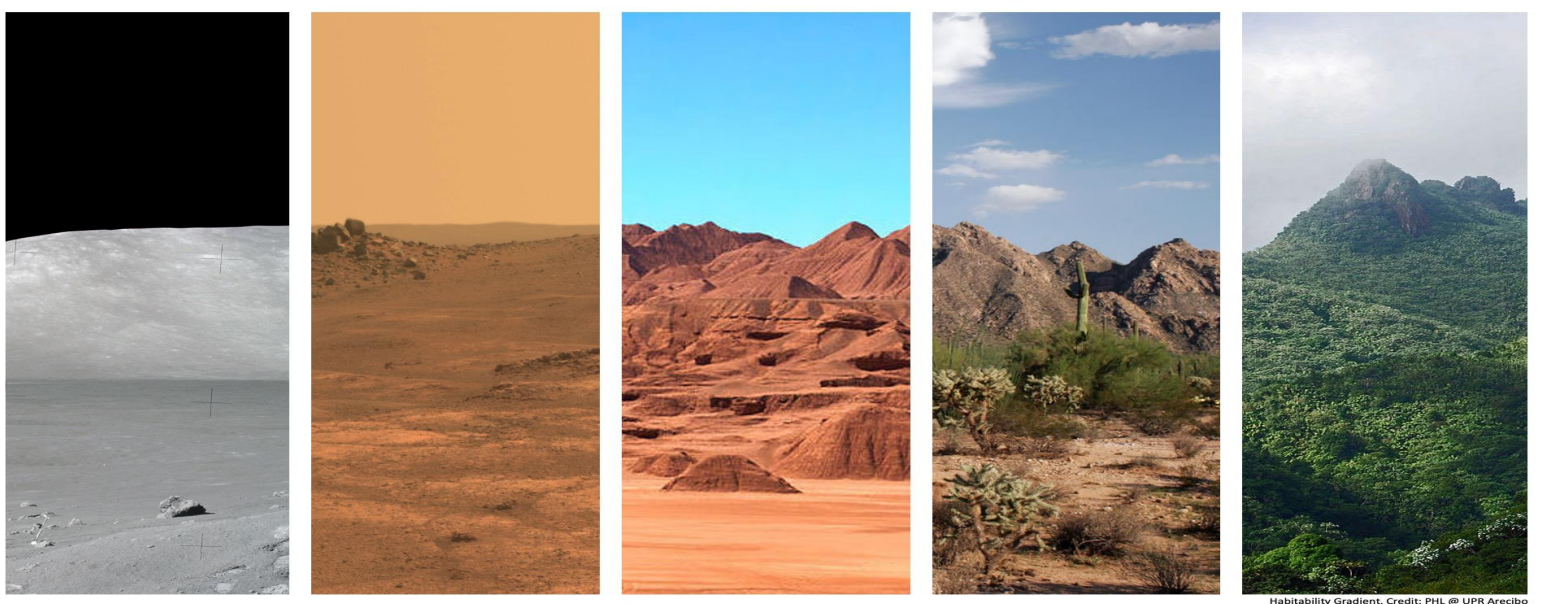

Abel Méndez ${ }^{1}$, Edgard G. Rivera-Valentín², Dirk Schulze-Makuch ${ }^{3,27,28}$, Justin Filiberto², Ramses Ramírez ${ }^{4,29}$, Tana E. Wood $^{5}$, Alfonso Dávila ${ }^{6}$, Chris McKay ${ }^{6}$, Kevin Ortiz Ceballos ${ }^{1}$, Marcos Jusino-Maldonado ${ }^{1}$, Nicole Torres Santiago ${ }^{1}$, Guillermo Nery ${ }^{1}$, René Heller ${ }^{7}{ }^{30}$, Paul Byrne ${ }^{8}$, Michael J. Malaska ${ }^{9}$, Erica Nathan ${ }^{10}$, Marta Filipa Simões ${ }^{11}$, André Antunes $^{11}$, Jesús Martínez-Frías ${ }^{12}$, Ludmila Carone ${ }^{13}$, Noam R. Izenberg ${ }^{14}$, Dimitra Atri ${ }^{15}$, Humberto Itic Carvajal Chitty $^{16}$, Priscilla Nowajewski-Barra ${ }^{17}$, Frances Rivera-Hernández ${ }^{18}$, Corine Brown ${ }^{19}$, Kennda Lynch ${ }^{2}$, David C. Catling ${ }^{20}$, Jorge I. Zuluaga ${ }^{21}$, Juan F. Salazar ${ }^{22}$, Howard Chen ${ }^{23}$, Grizelle González ${ }^{5}$, Madhu Kashyap Jagadeesh ${ }^{24}$, Jacob Haqq-Misra $^{25}$, Rory Barnes ${ }^{20}$, Charles S. Cockell ${ }^{26}$

${ }^{1}$ Planetary Habitability Laboratory, University of Puerto Rico at Arecibo, Puerto Rico, USA; ${ }^{2}$ Lunar and Planetary Institute, USRA, USA; ${ }^{3}$ Center for Astronomy and Astrophysics, Technische Universität Berlin, Germany; ${ }^{4}$ Earth-Life Science Institute, Japan; ${ }^{5}$ International Institute of Tropical Forestry, USDA Forest Service, Puerto Rico, USA; ${ }^{6}$ NASA Ames Research Center, USA; ${ }^{7}$ Max Planck Institute for Solar System Research, Germany; ${ }^{8}$ North Carolina State University, USA; ${ }^{9}$ Jet Propulsion Laboratory / California Institute of Technology, USA; ${ }^{10}$ Brown University, USA; ${ }^{11}$ State Key Laboratory of Lunar and Planetary Sciences, Macau University of Science and Technology, China; ${ }^{12}$ Instituto de Geociencias (CSIC-UCM), Spain; ${ }^{13}$ Max Planck Institute for Astronomy, Germany; ${ }^{14}$ Johns Hopkins Applied Physics Laboratory, USA; ${ }^{15}$ Center for Space Science, New York University Abu Dhabi, United Arab Emirates; ${ }^{16}$ Universidad Simón Bolívar, Venezuela; ${ }^{17}$ Fundación Ciencias Planetarias, Chile; ${ }^{18}$ Georgia Institute of Technology, USA; ${ }^{19}$ Macquarie University, Australia; ${ }^{20}$ University of Washington, USA; ${ }^{21}$ Institute of Physics / FCEN - Universidad de Antioquia, Colombia; ${ }^{22}$ GIGA, Escuela Ambiental, Facultad de Ingeniería, Universidad de Antioquia, Colombia; ${ }^{23}$ Center for Interdisciplinary Exploration and Research in Astrophysics, Northwestern University, USA; ${ }^{24}$ Jyoti Nivas College, Bengaluru, India; ${ }^{25}$ Blue Marble Space Institute of Science, USA; ${ }^{26}$ UK Centre for Astrobiology, UK; ${ }^{27}$ German Research Centre for Geosciences (GFZ), Section Geomicrobiology, Germany; ${ }^{28}$ Department of Experimental Limnology, Leibniz-Institute of Freshwater Ecology and Inland Fisheries (IGB), Germany; ${ }^{29}$ Space Science Institute, Japan; ${ }^{30}$ University of Göttingen, Institute for Astrophysics, Germany.

\section{Abstract}

Habitability has been generally defined as the capability of an environment to support life. Ecologists have been using Habitat Suitability Models (HSMs) for more than four decades to study the habitability of Earth from local to global scales. Astrobiologists have been proposing different habitability models for some time, with little integration and consistency between them and different in function to those used by ecologists. In this white paper, we suggest a mass-energy habitability model as an example of how to adapt and expand the models used by ecologists to the astrobiology field. We propose to implement these models into a NASA Habitability Standard (NHS) to standardize the habitability objectives of planetary missions. These standards will help to compare and characterize potentially habitable environments, prioritize target selections, and study correlations between habitability and biosignatures. Habitability models are the foundation of planetary habitability science. The synergy between the methods used by ecologists and astrobiologists will help to integrate and expand our understanding of the habitability of Earth, the Solar System, and exoplanets.

Corresponding Author: Abel Méndez (abel.mendez@upr.edu)

An extended version of this white paper is available at https://arxiv.org/abs/2007.05491

A list of endorsers is available at http://phl.upr.edu/librarv/notes/habmodels 


\section{Introduction}

Life on Earth is not equally distributed. There is a measurable gradient in the abundance and diversity of life from deserts to rain forests (i.e., spatially), and temporally among seasons and, at large time-scales, in geological time. Our planet also has experienced global environmental changes from the Archean to the Anthropocene. In general, a habitable environment is a spatial region that might support some form of life (Farmer, 2018), albeit not necessarily one with life. One of the biggest problems in astrobiology is how to define and measure the habitability not only of terrestrial environments but also of planetary environments, from the Solar System to extrasolar planets. The word Habitability literally means the quality of habitat (the suffix -ity means quality, state, or condition). Astrobiologists have been constructing different general definitions of habitability, not necessarily consistent with one another, for some time (e.g., Shock \& Holland, 2007; Hoehler, 2007; Cardenas et al., 2014; Cockell et al., 2016; Cárdenas et al., 2019; Heller, 2020). Other more specific habitability definitions, such as the canonical Habitable Zone (i.e., presence of surface liquid water on Earth-like planets), are used in exoplanet science (Kasting et al., 1993). Ecologists developed a standardized system for defining and measuring habitability in the early 1980s; however, this is seldom utilized in the astrobiology community (USFWS, 1980).

The popular term habitability is formally known as habitat suitability in biology. Ecologists before the 1980s were using different and conflicting measures of habitability, a situation not much different than today for astrobiologists. The U.S. Fish and Wildlife Service (USFWS) decided to solve this problem with the development of the Habitat Evaluation Procedures (HEP) standards in 1974 for use in impact assessment and project planning (USFWS, 1980). These procedures include the development and application of Habitat Suitability Models (HSM) (Hirzel \& Lay, 2008). Other names for these models are Ecological Niche Models (ENM), Species Distribution Models (SDMs), Habitat Distribution Models (HDM), Climate Envelope Models (CEM), Resource Selection Functions (RSF), and many other minor variants (Guisan et al., 2017). These multivariate statistical models are widely used today by ecologists to quantify species-environment relationships from the ground to satellite observations. Habitat Suitability Models integrate concepts as needed from ecophysiology, niche theory, population dynamics, macroecology, biogeography, and the metabolic theory of ecology.

Astrobiologists have largely not utilized HSMs for at least three reasons. First is the naming: habitability is a common word in Earth and Planetary Science, but it is not generally used by biologists. Thus, a quick review of the scientific literature shows no definition of this concept in biological terms. The second reason is the specialization: HSM is a specialized topic of theoretical ecology, which is not highly represented in the astrobiology community. The third is applicability: HSMs are mostly used to study the distribution of wild animals and plants, not microbial communities (generally the focus of astrobiological studies), so it may not seem readily applicable to the field of astrobiology. Yet endosymbiotic relationships between microorganisms (bacteria, fungi and other unicellular life) with animals and plants also play a key role in the survival of the latter. Thus, anything that can be said about habitability at the macroscopic level is tightly coupled to habitability at the microscopic level. In one way, the mathematical framework behind HSM is easier to apply to microbial communities than animals 
because the spatial interactions of animals (e.g., predation) tend to be much more complex. However, microbial life is not easy to quantify in free-living populations and it is thus harder to validate the HSMs with them, although molecular methods are changing this (Douglas, 2018).

Here we recommend adapting and expanding the ecologists' nearly four decades of experience modeling habitability on Earth to astrobiological studies. These models can be used to characterize the spatial and temporal distribution of habitable environments, identify regions of interest in the search for life, and, eventually, explore correlations between habitability and biosignatures. For example, such models would help to test the hypothesis that biosignatures (or biomarkers) are positively correlated with proxy indicators of geologically habitable environments (or geomarkers); i.e., there is life whenever there are habitable environments on Earth (Martinez-Frias et al., 2007). Measurements by past and future planetary missions can be combined into a standard library of habitability models. Results from different missions can then be compared, even using different measurements, since, through the use of HSMs, their results can be mapped to the same standard scale (e.g., zero for worst and one for best regions). A Habitability Readiness Analysis (HRA) of any mission could be used to determine how its existing instruments could be used, or what sensors should be added, for measurements in the spatial and temporal habitability scales of interest. Furthermore, it might also be possible to develop new sensors for direct habitability measurements.

This white paper to the Planetary Science and Astrobiology Decadal Survey 2023-2032 addresses many of the misconceptions about habitability and attempts to create a standard conceptual framework to assess habitability issues for future purposes. This contribution is relevant for roving, landed, or orbital missions for any planetary target of astrobiological interest, including environments on Earth. Section 2 describes our proposed general mass-energy approach to model habitability. Section 3 presents our specific recommendations to the Decadal Survey. Section 4 proposes science questions to be addressed by these models, beyond those traditionally associated with habitability studies. Finally, Section 5 presents our concluding remarks. The extended version of this white paper (Méndez et al., 2020) has an overview of current ecology models, how similar models are implemented in the astrobiology field, and a specific example of the application of our mass-energy habitability model to the Martian surface.

\section{A General Mass-Energy Model for Habitability}

The classical elements of air (gas), water (liquid), earth (solid), and fire (energy) are a powerful analogy to understand habitability. In essence, life needs environments where the three phases of matter and energy coexist. The five elements that constitute RNA and DNA ( $H, C, N, O$, and $P$ ) are not all available in a single phase of matter. For example, under temperate conditions, nitrogen is more likely available in a gas phase, whereas inorganic sulfur is widely available in rocks as sulfates. The dissolution, diffusion, and flow capabilities of air and water allow all these elements to mix and be readily available in any habitat. Following this analogy, when a product of life, such as wood, is burned it releases all the classical elements back to the environment: gas (e.g., $\mathrm{CO}_{2}$ ), liquid (water), solid (ashes), and energy (heat). On a planetary scale, the classical elements become the atmosphere, hydrosphere, lithosphere - the habitable trinity of Dohm \& Maruyama (2015) - plus photosynthetic or chemosynthetic energy. Thus, habitability depends 
fundamentally on the availability of raw materials and energy to assemble and maintain life, among other factors (e.g., decay and recycling of matter).

The analogy between habitability and the classical elements can be formalized into a general quantitative framework. Here we propose that habitability is proportional to the mass and energy available for life. This definition is consistent with the habitability models developed in ecology (i.e., proportional to the carrying capacity) and explicitly includes energy as suggested by others (Hoehler, 2007; Macalady et al., 2013). Our model is applicable to any type of environment, from microenvironments to entire biospheres. The main challenge is to convert the intensive or extensive properties of interest (e.g., temperature and water activity) to quantities proportional to the mass and energy of the environment. The mass available for life is usually a small fraction of the total environment mass and is further constrained by any limiting ingredient (e.g., nutrients). For example, having more water does not make oceans more habitable, as life is limited by the availability of iron and sulfur in them. In fact, high concentrations of some ingredients might be harmful because they dilute other essential ingredients or make the environment toxic. Negative factors, like ionizing radiation, reduce the fraction of the available mass and energy (e.g., Atri, 2017).

Our proposed habitability model can be constructed in six steps: (1) select the space and time of the region of interest (i.e., define the boundary conditions); (2) select variables and convert them to quantities proportional to mass or energy; (3) select species or communities and their ecophysiological response curves for the selected variables; (4) identify one or more standards of comparison (i.e., a terrestrial or planetary analog); and (5) solve the habitability master equation (Méndez et al., in preparation):

$$
\frac{\partial^{2} H}{\partial s \partial t}=\left(\frac{1}{M} \frac{\partial^{2} M}{\partial s \partial t}+\frac{1}{E} \frac{\partial^{2} E}{\partial s \partial t}\right) H+\frac{\partial M}{\partial s} \frac{\partial E}{\partial t}+\frac{\partial M}{\partial t} \frac{\partial E}{\partial s}
$$

where $H$ is the habitability, $M$ and $E$ are the normalized mass and energy available (i.e., a fraction of the total mass and energy) for the life of interest (i.e., a species or community) relative to the standard of comparison, respectively, and $s$ and $t$ are the spatial (e.g., area or volume) and temporal (e.g., hours or days) components. The last step, then, is to (6) validate and correct the habitability model with environments on Earth, if possible (i.e., find positive correlations between habitability and biomass, productivity, or biosignatures). For example, equation 1 can be used to compare the habitability of a specific volume of ocean water of Europa relative to the same volume of deep ocean waters on Earth, given mass and energy fluxes. Sometimes it is not desirable to use the same volume or time periods for comparison purposes (e.g., comparing early Mars with contemporary Earth). The space of interest is not limited by planetary scales. It can be enclosing a stellar region to evaluate its overall habitability, e.g., a galactic habitable zone (Spitoni et al., 2017). The general population growth equations (e.g., exponential and logistic) can also be derived from equation 1.

The construction of a habitability model is not easy. Our model provides at least an upper limit for the habitability of a system for a given set of parameters, and is further improved by properly selecting key environmental variables and ecophysiological response curves for the 
life of interest (steps 2 and 3). Any model must be validated with environments on Earth where a positive correlation between habitability and the presence or abundance of life should be observed (step 6). The simple solution of equation 1 is $H=M E$ (units of kgJ), or more practically, $H^{\prime}=\rho P$ (units of $\mathrm{Wkgm}^{-3}$ ), where $H^{\prime}$ is the specific habitability, $\rho$ is the concentration of one or more ingredients necessary for life, and $P$ is the available metabolic power (assuming that mass only depends on space and energy on time). In practice, each of these variables could be normalized to the standard of comparison for simplicity and consistency, where zero denotes a non-habitable environment and one denotes a highly habitable environment. Also, occurrence or probabilities could be used instead of these variables, which is exactly what the definition of the Habitable Zone does (i.e., probability of surface water $\geq 0$ ). Negative values could be used to quantify the damaging effect of non-habitable environments (e.g., comparing the surface of the Moon and Venus). Values larger than one could represent super-habitable conditions relative to a standard. An example of the application of these models is available in Méndez et al. (2020).

\section{Recommendations for Planetary Exploration Missions}

Planetary exploration missions are playing a critical role in our understanding of planetary habitability beyond what remote sensing from space can provide. The habitability of particular environments on planets such as Mars or Europa can be explored and compared thanks to targeted measurements taken with multiple orbital and ground sensors (e.g., temperature, radiation, etc.), with which habitability models can be constructed. At the same time, future mission designs can synergistically take advantage of the predictions of habitability models in their selection of potential exploration strategies, mission priorities, and instruments, whether they are primarily astrobiological missions or not. Planetary exploration mission designs for the upcoming decades may have major astrobiological components that can directly or indirectly inform the study of habitability in the Solar System - even if the determination of habitability is not the primary focus of a mission. Indeed, general mission components not directly designed for astrobiological purposes might usefully contribute to habitability studies with only minimal considerations in design. Here we list three recommendations for the planetary community:

1. Increase and widen the participation of more experts on habitat suitability models. Ecologists are the experts in the ground-truthed proven measurement of terrestrial habitability, yet they are seldom represented in the planetary and astrobiology community. New synergies between NASA and the national and international ecological societies, e.g., the Ecological Society of America (ESA), Soil Ecology Society (SES), and the International Society for Microbial Ecology (ISME), should be established via, for example, a joint conference session at the Lunar and Planetary Science Conference. There should be worldwide participation to guarantee global standardization. This synergy will stimulate the participation and exploration of the Solar System as a laboratory for expanding our current understanding of the habitability of Earth.

2. Further terrestrial exploration. Many Earth habitats are vastly under-explored biologically. For example, the clouds, stratosphere, deep ocean, deep ice, deep earth, or the mantle (e.g., Lollar et al., 2019; DasSarma et al., 2020). Further, astrobiology needs to make stronger connections to the researchers working in these under-studied environments (e.g., The Deep Carbon Observatory) so that there is a cohesive understanding of the 
state-of-the-art science being learned and efforts to continue to study these environments are supported. These field studies should provide new data to test the applicability of current habitability models with extreme environments, and thus get us closer to diverse planetary conditions. At the same time, unicellular life continually surprises us with new ways to survive and obtain energy from its environment (rock-eaters, electric currents, and even radioactivity) which shows us we need to be flexible in considering energy sources for habitability.

3. Improve habitability models. New habitability models should be developed and validated with field and laboratory experiments, including simulated extreme and planetary analog environments (e.g., Taubner et al., 2020). The main goal is to identify knowledge gaps. For example, new ecophysiological response curves (e.g., growth rate as a function of water activity, a measure of available water) for some organisms are necessary, especially in dynamic environments such as gradient-rich biotopes and higher complexity extreme environments (i.e., those with multiple extremes such as deep-sea brines). Also, there are insufficient models on microbial growth in near-surface dynamic environments (e.g., as applicable to martian diurnal cycles). There is a growing body of literature about the manifold mechanisms through which life affects the Earth's climate system, including the global energy balance and atmospheric composition and dynamics. Advances in the understanding of climate-life interactions in the Earth System (e.g. Bonan and Doney, 2018) can provide new insights for habitability models.

4. Develop a NASA Habitability Standard (NHS). Existing and future planetary missions should specify how they assess habitability for each of their instruments according to a shared NASA habitability standard. For example, measurements of surface temperature and water vapor from landers or orbital missions could be converted into a simple habitability model. The advantage of a standard is that past and future missions could be compared to each other and their habitability assessments refined, and new habitability knowledge gaps could be identified. This dynamic standard should be evaluated and updated regularly by a diverse and multidisciplinary committee, for example during a Decadal Survey and/or mid-decade review. Currently, the closest concept to an NHS is specific language included in various NASA roadmaps, such as the NASA Astrobiology Roadmap (Des Marais et al., 2008) and the NASA Roadmap to Ocean Worlds (Hendrix et al., 2018). These documents stress the need for habitability evaluations and missions (e.g., Europa Clipper and Titan Dragonfly), yet only focus on the individual habitability requirements and not how to combine the net contribution of these factors. Furthermore, the NHS might eventually become the standard of other disciplines.

\section{Science Questions}

Each astrobiological relevant planetary mission should answer a series of basic scientific questions about the environment(s) to be studied as a core part of the planning process. The answers to these questions should be updated based on mission results. To do so, it is important to define an environment of interest, both in space and time (termed a quadrat in ecology), and anticipate the following science questions as part of the initial analysis: 
1. What are the limiting factors? Usually, there is a small set of main factors (e.g., edaphic factors) that influence living organisms (e.g., water, nutrients). These will be the first set of variables to be used for the construction of a habitability model, which will later be refined with more variables. For example, primary productivity is mainly driven by temperature, precipitation, and nutrients on land, and by temperature and nutrient concentrations in the oceans, among other factors. In general, these factors should be directly or indirectly related to the mass and energy of the environment (e.g., Martiny et al., 2006; Pikuta et al., 2007; Williams \& Hallsworth, 2009; Harrison et al., 2013; McKay, 2014; Lynch \& Neufeld, 2015; Tecon \& Or, 2017).

2. What are the terrestrial and planetary analogs? Identify at least one analog on Earth and one close planetary analog as the comparison standards (i.e., for model normalizations). For example, if studying a particular martian environment, select the terrestrial polar deserts and a martian analog based on the variables of interest. The cross-comparison of similar types of environment (e.g., salterns), as well as slightly different settings (e.g., high salinity biotopes with different $\mathrm{pH}$, temperature, or chemical conditions), could also prove useful. The subsurface oceans of Europa or Enceladus could be compared with deep seawater, hydrothermal systems, or deep-sea brines (Antunes et al., 2020). Planetary atmospheres could be compared with high altitude or near-space regions. An analysis of similarities (e.g., ANOSIM) could be used to formally select and compare these regions (Clarke, 1993).

3. What is the habitability value? The habitability of the region of interest is evaluated based on the selected environmental factors, and then compared with the selected Earth and planetary analogs, using a normalized scale from zero to one for simplicity. A library of habitability measures is usually constructed (i.e., a habitability matrix), each for different considerations (e.g., species). These inputs are then used to construct multivariate habitability maps (niche quantification in ecology) for site selections.

4. What is the potential biomass? The upper limits of biomass can be predicted based on the fluxes of mass and energy available for life, and usually a very small fraction of the total mass and energy. For example, biomass could be estimated from the available metabolic energy using the Metabolic Theory of Ecology (van der Meer, 2006; Schramski et al., 2015; Clarke, 2017). These upper limits are used in the sensitivity designs of life detection experiments. Available free energy from known disequilibria has been used to estimate an upper limit on the biomass in the subsurface of Mars and its value depends on uncertainties of the abundances of metabolic reactants and the assumed microbial basal power requirement (Sholes et al., 2019).

5. What is the expected correlation between habitability and biosignatures? The potential upper values of biomass can be converted to estimates of observable biosignatures or disequilibrium chemistry (Catling et al., 2018). Habitability and biosignatures are positively correlated on Earth but this might not be necessarily true for other planets. A zero or negative correlation could indicate an incorrect habitability model or a biological process unlike Earth (life as we don't know it). The habitability-biosignatures correlation is a fundamental problem of astrobiology, but non-detections are also important. For example, 
it will be profound to detect planetary regions determined to be habitable by Earth standards yet devoid of any life. Such discoveries would place bounds on abiogenesis.

\section{Conclusion}

Habitability models are successful analysis tools for characterizing habitable environments on Earth. Ecologists have been using these models for more than four decades to understand the distribution of terrestrial life at local to global scales. Astrobiologists have been proposing different models for some time, with little integration and consistency between them and different in function to those used by biologists. In this white paper, we suggest a mass-energy habitability model as an example of how to adapt and expand the models used by ecologists to the astrobiology field. Our model could be used to compare environments and prioritize targets for exploration. NASA should create habitability standards for planetary missions with astrobiology objectives, as the USFWS successfully did long ago for ecologists. These standards are necessary to make sense of data from multiple missions, develop predictions for environmental niches on planetary bodies that can be tested, and understand the extraterrestrial correlations between habitability and biosignatures.

There is no need for the planetary and astrobiology community to reinvent the methods and tools used by ecologists. It is true now that the ecology methods are more capable than our limited planetary data allows, but they provide the basic language and framework to connect Earth and planetary sciences for decades to come. For example, there are many theoretical and computational tools used in ecology to quantify environments and their habitability, mostly known as habitat suitability models. See Guisan et al. (2017) for an extensive review of these models and Lortie et al. (2020) for a current review of the computational tools. Most of these tools are available as packages in the $\underline{\mathrm{R} \text { Computing Language in the Comprehensive R Archive }}$ Network (CRAN) and GitHub (e.g., Environmetrics, HSDM). New, higher-resolution remote sensing instruments and exploration technologies will create better habitability maps from rover, lander, and orbiter data. Habitability models will eventually lead us to a better understanding of the potential for life in the Solar System and beyond, and perhaps even the factors that influence the development of life itself. Habitability models are the foundation of planetary habitability science. After all of our scientific and technological advances, we still need a stronger integration between biology, planetary sciences, and astronomy (Cockell, 2020).

References (available in the extended version of this white paper below)

Méndez, A., Rivera-Valentín, E. G., Schulze-Makuch, D., Filiberto, J., Ramírez, R., Wood, T. E., Dávila, A., McKay, C., Ortiz Ceballos, K., Jusino-Maldonado, M., Nery, G., Heller, R., Byrne, P., Malaska, M. J., Nathan, E., Filipa Simões, M., Antunes, A., Martínez-Frías, J., Carone, L., ... Barnes, R. (2020). Habitability Models for Planetary Sciences. ArXiv E-Prints, 2007, arXiv:2007.05491. https://arxiv.org/abs/2007.05491

This work was supported by the NASA Astrobiology Institute (NAI), the Planetary Habitability Laboratory (PHL), and the University of Puerto Rico at Arecibo (UPR Arecibo). Thanks to NASA Puerto Rico Space Grant Consortium and the Puerto Rico Louis Stokes Alliance For Minority Participation (PR-LSAMP) for supporting our students. Thanks to Ravi kumar Kopparapu from the NASA Goddard Space Flight Center for valuable comments. 